edpsciences IOP Institute of Physics

\section{Related content}

- Modelling the effect of randomly dispersed
$\frac{\text { adatoms on carbon nanotubes }}{\text { C G Rocha, A Wall, A R Rocha et al. }}$
- The influence of Gaussian strain on
$\frac{\text { sublattice selectivity of impurities in }}{\text { graphene }}$
James A Lawlor, Claudia G Rocha,
Vanessa Torres et al.
- Local density of states and scanning
$\frac{\text { tunneling currents in graphene }}{\text { N M R Peres, Ling Yang and Shan-Wen }}$
Tsai

View the article online for updates and enhancements.

Commensurability effect on the electronic structure of carbon nanostructures: Impact on supercell calculations in nanotubes

To cite this article: M. S. Ferreira et al 2017 EPL 11727005

\section{Recent citations}

$\frac{\text { Finite-size correction scheme for supercell }}{\text { calculations in Dirac-point two-dimensional }}$
materials
C. G. Rocha et al 


\title{
Commensurability effect on the electronic structure of carbon nanostructures: Impact on supercell calculations in nanotubes
}

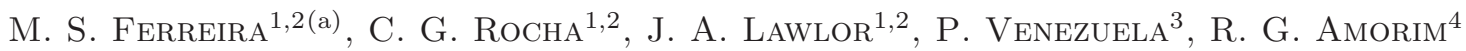 \\ and A. R. RoCHA ${ }^{5,6}$ \\ 1 School of Physics, Trinity College Dublin - Dublin 2, Ireland \\ 2 CRANN, Trinity College Dublin - Dublin 2, Ireland \\ 3 Instituto de Física, Universidade Federal Fluminense - Niterói, RJ, Brazil \\ 4 Departamento de Fisica, ICEx, Universidade Federal Fluminense - Volta Redonda, RJ, Brazil \\ 5 Instituto de Física Teórica, Universidade Estadual Paulista (Unesp) - São Paulo, SP, Brazil \\ ${ }^{6}$ Department of Chemical Engineering, Massachusetts Institute of Technology - Cambridge, MA, USA
}

received 13 December 2016; accepted in final form 20 February 2017

published online 9 March 2017

PACS 71.15.-m - Methods of electronic structure calculations

PACS 73.63.Fg - Nanotubes

PACS 73.22.Pr - Electronic structure of graphene

\begin{abstract}
Friedel oscillations are ubiquitous features seen in all impurity-doped metallic structures but in the case of graphene-like materials they are not so evident because the relevant wavelengths are perfectly commensurate with the lattice parameter. Here we demonstrate that this commensurability effect leads to a slow convergence of supercell-based total energy calculations in impurity-doped carbon nanotubes. We derive a mathematically transparent expression for the formation energy and identify a very distinctive dependence on the size of the supercell unit. We make use of this dependence through a simple extrapolation scheme to obtain density functional theory results with accuracy levels that would otherwise require enormously large unit cells.
\end{abstract}

Copyright (C) EPLA, 2017

Introduction. - One of the signatures of a symmetrybreaking perturbation in solids is the appearance of spatial fluctuations in quantities such as the local density of states (LDOS) and the carrier density, both of which oscillate away from the perturbation. Commonly known as Friedel oscillations (FO) [1], these are seen in all impuritydoped metallic systems. In the case of hosts made of either graphene $[2,3]$ or its derivatives [4], such oscillations are hidden by a simple commensurability effect that suppresses this otherwise ubiquitous feature [5]. Because the characteristic wavelengths are identical to the spacing between equivalent carbon sites, one has the impression that there are no FO in graphene-based materials [6].

This apparently innocuous effect has an impact on several fronts. For a start, it explains the sublattice asymmetry seen in impurity-doped graphene where dopants favour one of the two sublattices of graphene even though both are absolutely equivalent [7-9]. Originally seen in graphene substitutionally doped with nitrogen [10,11],

(a) E-mail: ferreirmtcd.ie sublattice asymmetry has also been reported in graphene samples adsorbed with hydrogen [12], molybdenum [13] and boron [14]. Another consequence of having local fluctuations with exactly the same wavelength as the carboncarbon spacing is that the bonding symmetry with which a dopant binds to graphene may determine how strong a scatterer this dopant is. As a result, centre-bonded impurities tend to be completely invisible causing no scattering whatsoever $[15,16]$. In addition, the commensurability effect is again the key behind the prediction that magnetic impurities couple ferromagnetically or antiferromagnetically depending exclusively on whether they are located on the same or on distinct sublattices [6,17-20]. Finally, another important consequence of this effect is the extra care needed for calculations of magnetically doped graphene when there is a natural tendency for anti-parallel alignment between the magnetic moments [21,22].

One simple way of explaining the existence of such a commensurability effect is to remember that dopantinduced FO are determined by the geometry of the host's Fermi surface. In the case of graphene-based materials, 
the Fermi surface consists of six points on the corners of the hexagonal Brillouin zone (BZ). In this case, the presence of a symmetry-breaking dopant generates FO with wave vectors that span the $\mathrm{BZ}$ and when that happens the corresponding wavelengths become identical to the periodicity of the underlying lattice. In other words, FO are perfectly commensurate with the lattice parameter of graphene simply because of the peculiar Fermi surface of this material.

Here we demonstrate another important case in which the commensurability effect in graphene-based materials may have a very strong repercussion, this time on improving the accuracy of total energy electronic structure calculations that are carried out in the so-called supercell representation. It is worth remembering that most density functional theory (DFT) [23,24] implementations are indeed based on this representation and for that reason will serve as the main testbed of our study. We shall focus on carbon nanotubes (CNT) because the impact of the commensurability effect is more pronounced as the system dimensionality is reduced [25].

General theory. - Let us start by defining the model Hamiltonian that will guide us throughout the manuscript. The system consists of a single substitutional impurity inserted into an otherwise crystalline carbon nanotube. The system is fully described by the Hamiltonian $\hat{H}=\hat{H}_{0}+\hat{V}$, where

$$
\hat{H}_{0}=\sum_{\langle i, j\rangle}|i\rangle t_{i, j}\langle j|
$$

corresponds to the pristine nearest-neighbour tightbinding Hamiltonian of the nanotube and the operator $\hat{V}=|0\rangle \lambda\langle 0|$ represents a mere shift in the on-site potential where the impurity is located (site $i=0$ ). The matrix elements $t_{i, j}=t$ when $i$ and $j$ label nearest-neighbour sites and vanish otherwise. The value of $t$ is arbitrary and will hereafter serve as our energy unit. It is worth emphasizing that the simplicity of this single-orbital Hamiltonian is in no way a limiting factor since it can be easily generalized to a multi-orbital representation which may account for further nearest-neighbour couplings as well. Likewise, the premise that the introduction of a substitutional impurity impacts only the on-site potential on the impurity site is a consequence of the screening and can be easily relaxed without any qualitative change to our results and conclusions.

Associated with the Hamiltonian $\hat{H}_{0}$, we assign the single-particle Green function (GF) defined as $\hat{\mathcal{G}}=(E-$ $\left.\hat{H}_{0}\right)^{-1}$, being $E$ the energy. The LDOS $\rho_{\ell}$ at an arbitrary site $\ell$ can be easily expressed in terms of $\hat{\mathcal{G}}$ as follows:

$$
\rho_{\ell}=-\frac{1}{\pi}\left\{\operatorname{Im}\left[\mathcal{G}_{\ell, \ell}\right]+\operatorname{Im}\left[\mathcal{G}_{\ell, 0} \lambda\left(1-\mathcal{G}_{0,0} \lambda\right)^{-1} \mathcal{G}_{0, \ell}\right]\right\},
$$

where the first term on the r.h.s of the equation above represents the LDOS of the impurity-free system. Note that the position dependence of the LDOS in eq. (2) is entirely contained in the product $\mathcal{G}_{0, \ell} \times \mathcal{G}_{\ell, 0}$. This position dependence is bound to manifest itself in other quantities as well, one of them being the integrated LDOS, i.e., the charge density.

Rather than writing the charge density itself, it is convenient to express the change in this quantity as a result of the introduction of the impurity and how it depends on the LDOS variation. It is given by

$$
\Delta n_{\ell}=\int_{-\infty}^{+\infty} \mathrm{d} E f(E) \Delta \rho_{\ell},
$$

where $f(E)$ is the Fermi-Dirac distribution function and the quantities $\Delta n_{\ell}$ and $\Delta \rho_{\ell}$ correspond to the changes in charge density and in LDOS at site $\ell$, respectively.

In addition, the energy cost associated with the introduction of the impurity can be expressed as the change in total energy, i.e.,

$$
\Delta \mathcal{E}_{1}=\sum_{\ell} \int_{-\infty}^{+\infty} \mathrm{d} E f(E) E \Delta \rho_{\ell},
$$

where the subscript 1 refers to the introduction of a single impurity. $\Delta \mathcal{E}_{1}$ is associated with the energy-band contribution to the total energy variation and is present in quantities like the binding energy and formation energy, for instance. Fortunately, there are certain sum rules that permit eq. (4) to be rewritten in a far more convenient form, namely [7]

$$
\Delta \mathcal{E}_{1}=\frac{1}{\pi} \int_{-\infty}^{+\infty} \mathrm{d} E f(E) \operatorname{Im} \ln \left[1-\mathcal{G}_{0,0} \lambda\right] .
$$

Whereas $\Delta \rho_{\ell}$ obviously depends on $\ell$, the quantity $\Delta \mathcal{E}_{1}$ is not position dependent. Indeed, eq. (4) contains a sum over all sites $\ell$ and eq. (5) involves only the diagonal matrix element $\mathcal{G}_{0,0}$ which is identical to any other diagonal element $\mathcal{G}_{\ell, \ell}$ and therefore carries no dependence on position.

We now turn our attention to the energy cost $\Delta \mathcal{E}_{2}$ associated with the introduction of two impurities, one at site 0 and the other at site $\ell$ a distance $D$ apart. In this case the potential $\hat{V}$ simply acquires another term, i.e., $\hat{V}=|0\rangle \lambda\langle 0|+| \ell\rangle \lambda\langle\ell|$. The energy cost in this case is given by $[7]$

$$
\Delta \mathcal{E}_{2}=2 \Delta \mathcal{E}_{1}+C(D)
$$

where

$$
C(D)=\int_{-\infty}^{+\infty} \mathrm{d} E f(E) \operatorname{Im} \ln \left[1-\frac{\lambda^{2} \mathcal{G}_{0, \ell} \mathcal{G}_{\ell, 0}}{\left(1-\lambda \mathcal{G}_{0,0}\right)^{2}}\right] .
$$

Note that the only position-dependent part of the integrand above is contained in the product $\mathcal{G}_{0, \ell} \times \mathcal{G}_{\ell, 0}$, similarly to the case seen in eqs. (2) and (3). For that reason, the quantity $C(D)$ is expected to display the same position dependence as the charge density $\Delta n_{\ell}$.

To generalize the energy cost expression in eq. (6) beyond a single pair of impurities we define the energy cost $\Delta \mathcal{E}_{N}$ associated with the introduction of $N$ impurities,

$$
\Delta \mathcal{E}_{N}=N \Delta \mathcal{E}_{1}+\sum_{\ell, m} C\left(D_{\ell, m}\right),
$$


where the sum now runs over all possible impurity pairs and $D_{\ell, m}$ represents the separation between impurities at sites $\ell$ and $m$. Note that any contribution beyond the pairwise interaction terms contained in $C(D)$ have been neglected. It is worth pointing out that any ionic contribution contained in the relaxation around the impurity is very localized and somewhat similar whether one calculates $\Delta \mathcal{E}_{N} / N$ or $\Delta \mathcal{E}_{1}$.

In the case of hosts whose energy cost for adding impurities is described by an oscillatory function $C(D)$, the sum in eq. (8) is likely to be averaged out. Whether the impurities are orderly spaced or randomly distributed, whenever the function $C(D)$ is oscillatory the single-impurity contribution to the energy cost $\Delta \mathcal{E}_{N}$ will dominate over the impurity-interaction contributions, i.e., $\Delta \mathcal{E}_{N} \approx N \Delta \mathcal{E}_{1}$. Bearing in mind that the oscillatory form of $C(D)$ results from the existence of $\mathrm{FO}$, this behaviour is expected to be prevalent in most materials. However, as previously explained, FO are hidden by the commensurability effect in carbon because the FO wavelengths coincide exactly with the nanotube lattice parameter $[5,25]$. Therefore, this suggests that a large number of impurities inserted in a nanotube will impact the energy balance of the system because the sum on the r.h.s. of eq. (8) will not be averaged out but may yield a contribution comparable to $N \Delta \mathcal{E}_{1}$.

In order to proceed, we require a more quantitative description of the function $C(D)$. Lawlor et al. [5,8] have shown that $C(D)$ is in general oscillatory and obeys the following functional form $C(D) \propto \cos (Q D) / D^{\alpha}$, where $Q$ and $\alpha$ are constants that depend on the geometry of the Fermi surface and on the system dimensionality, respectively. Mathematically, the explanation for the absence of FO in graphene-related materials lies in the fact that the commensurability effect makes the product $Q D=2 \pi$, which suppresses the oscillatory character of the function $C(D)$. With that in mind, we must revisit the energetics of a graphene-related material with a large number of impurities. Described by eq. (8), the energy balance becomes

$$
\frac{\Delta \mathcal{E}_{N}}{N}=\Delta \mathcal{E}_{1}+\frac{1}{N} \sum_{\ell, m} \frac{\gamma}{\left(D_{\ell, m}\right)^{\alpha}},
$$

where $\frac{\Delta \mathcal{E}_{N}}{N}$ is the energy cost per impurity and $\gamma$ is a constant. Note that even though the individual terms in the summation may be substantially smaller than $\Delta \mathcal{E}_{1}$, their addition may add up to a sizable contribution because without oscillations all terms have the same sign. In this case the interaction between impurities can certainly impact the overall energy balance of the system, leading to $\Delta \mathcal{E}_{N} / N \neq \Delta \mathcal{E}_{1}$.

One striking consequence of the absence of FO is what happens when impurities are orderly distributed. Consider, for example, a carbon nanotube with substitutional impurities placed homogeneously along its length. Although it might not seem like a realistic setup, this is the typical spatial arrangement in the supercell representation commonly used in most $a b$ initio computational methods for materials [26-28]. The rationale behind the use of a supercell in the study of doped systems is that total energy evaluations converge when the cells are large and impurities are sufficiently far apart $[29,30]$.

In the case of an infinitely long nanotube doped with one impurity per unit cell of size $L$, the energy balance equation above can be rewritten as

$$
\frac{\Delta \mathcal{E}_{N}}{N}=\Delta \mathcal{E}_{1}+\sum_{m=1}^{+\infty} \frac{2 \gamma}{(m L)^{\alpha}},
$$

where the summation index $m$ is an integer running from 1 to $+\infty$ and the factor 2 accounts for the 1-dimensional symmetry of the host. By factoring the unit cell size $L$ out of the summation, the change in total energy per unit cell acquires a simple form given by

$$
\frac{\Delta \mathcal{E}_{N}}{N}=\Delta \mathcal{E}_{1}+\frac{\beta}{L^{\alpha}},
$$

where $\beta \equiv 2 \gamma \sum_{m} m^{-\alpha}$.

Equation (11) is the key result of this manuscript and its interpretation is as follows. When calculating total energy variations of impurity-doped systems within the supercell representation, the quantity that is usually obtained is the energy change per unit cell $\Delta \mathcal{E}_{N} / N$. This value is expected to coincide with the real quantity of interest, namely $\Delta \mathcal{E}_{1}$, because $\beta \approx 0$ in the limit of sufficiently large values of $L$. This is indeed the case for the vast majority of materials that display FO. However, $\beta \neq 0$ in the absence of FO and the calculation of $\Delta \mathcal{E}_{N} / N$ alone is not sufficient to give direct information about $\Delta \mathcal{E}_{1}$. In this case the last term on the r.h.s of eq. (11) must also be taken into account.

Far from invalidating the supercell methodology, we argue that the lack of FO offers a great opportunity to enhance the accuracy of such calculations. By making use of the functional form shown in eq. (11), a plot of $\Delta \mathcal{E}_{N} / N$ as a function of $L^{-\alpha}$ should generate a distinctive straight line with the slope described by $\beta$ and with the intercept given by $\Delta \mathcal{E}_{1}$.

DFT supercell calculations. - As previously anticipated, the findings based on the simplified Hamiltonian defined in eq. (1) are very robust and should be seen in more realistic Hamiltonians [31,32]. With that in mind, we perform DFT calculations of nanotubes doped with substitutional impurities. Our goal is to show that DFTevaluated results for $\Delta \mathcal{E}_{N} / N$ plotted as a function of $1 / L^{\alpha}$, where $L$ is the unit cell size, also give rise to a distinctive straight line. Consequently, we are able to obtain $\Delta \mathcal{E}_{1}$ by a simple extrapolation mechanism with calculations involving only relatively small unit cell sizes.

Figure 1 shows the different structures considered in our calculations. We use a $(5,5)$ carbon nanotube containing either a single substitutional nitrogen or boron atom. In all cases the length of the cell $L$ is varied from 


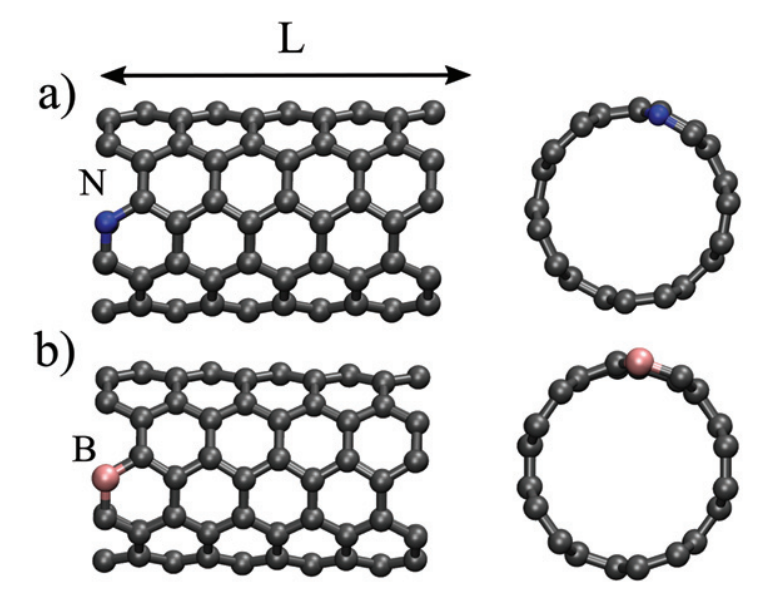

Fig. 1: (Color online) Top (left panels) and side (right panels) views of the structures considered in our calculations. A $(5,5)$ armchair CNT with a substitutional (a) nitrogen, or (b) boron impurity. In the figures, $L$ indicates the length of the unit cell.

1 to 15 nanotube rings. The electronic structure simulations were conducted with ab initio DFT as implemented in SIESTA [28]. We used the generalized gradient approximation (GGA-PBE) [33] for the exchange-correlation functional. The valence electrons are described by a double- $\zeta$ polarized (DZP) basis set, and norm-conserving pseudopotentials [34] were used. The cutoff for the real space grid was fixed at $450 \mathrm{Ry}$, and the number of $k$-points in the Brillouin zone varied depending on the size of the unit cell. For the smaller cell length $200 k$-points were used. In order to prevent spurious interaction between images a vacuum region of $20 \AA$ in the transverse direction was introduced. All structures were fully relaxed using a conjugate gradient (CG) algorithm with residual forces in each component of every atom smaller than $0.01 \mathrm{eV} / \AA$.

For comparing our DFT calculation with those of eq. (11) we consider the formation energy,

$$
E_{\mathrm{f}}(L)=E_{\text {def }}(L)-E_{\text {pris }}(L)+\sum_{i} n_{i} \mu_{i},
$$

where $E_{\text {pris }}(L)$ corresponds to the total energy of a pristine nanotube with supercell length $L, E_{\text {def }}(L)$ is the energy of its counterpart containing the impurity, $n_{i} \in \mathbb{N}$ is the number of atoms of species $i$ removed $\left(n_{i}>0\right)$ or added to the $\left(n_{i}<0\right)$ to the system, and finally $\mu_{i}$ is the chemical potential for the corresponding system. In the case of carbon, $\mu_{C}$ is the cohesive energy of a $(5,5)$ nanotube, and in the cases of nitrogen and boron, we considered the chemical potential as half of the binding energy for the corresponding diatomic molecule. Ultimately the choice of chemical potential is somewhat arbitrary and will only introduce a rigid shift on $E_{\mathrm{f}}$ as it is not dependent on the size of the system.

Results. - The formation energy $E_{\mathrm{f}}$ for a $(5,5)$ nanotube doped with $\mathrm{N}$ and with $\mathrm{B}$ can be seen in figs. 2 and 3, respectively. The top panels of both figures plot $E_{\mathrm{f}}$ as a
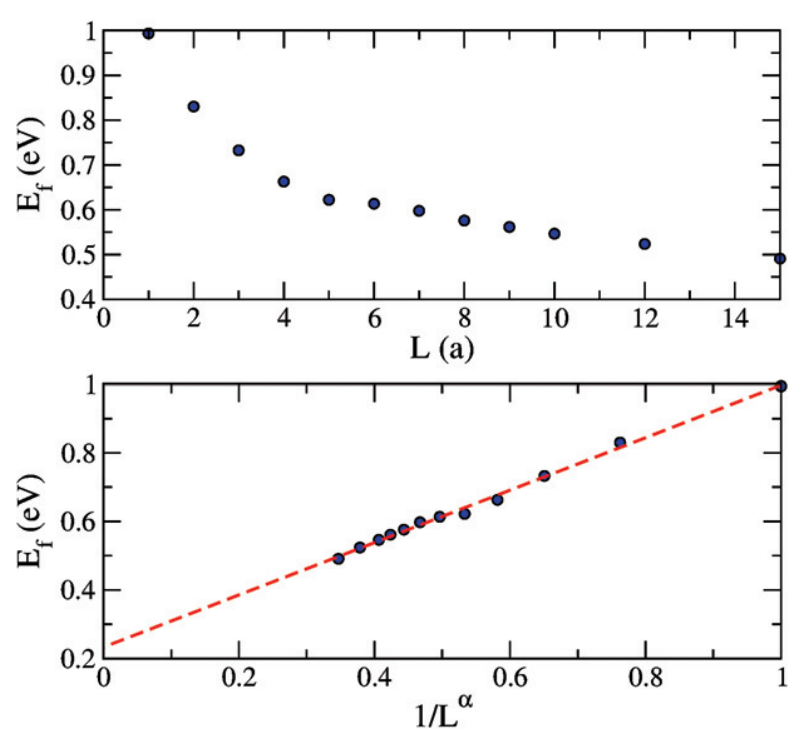

Fig. 2: (Color online) Formation energy for a carbon nanotube with nitrogen substitutional doping as a function of (top panel) unit cell size $L$ (in units of the graphene lattice parameter, $a$ ) and (bottom panel) $1 / L^{\alpha}$, with $\alpha=0.391$. The intercept of the linear function depicted by the dashed line gives $\Delta \mathcal{E}_{1}=$ $0.233 \mathrm{eV}$.
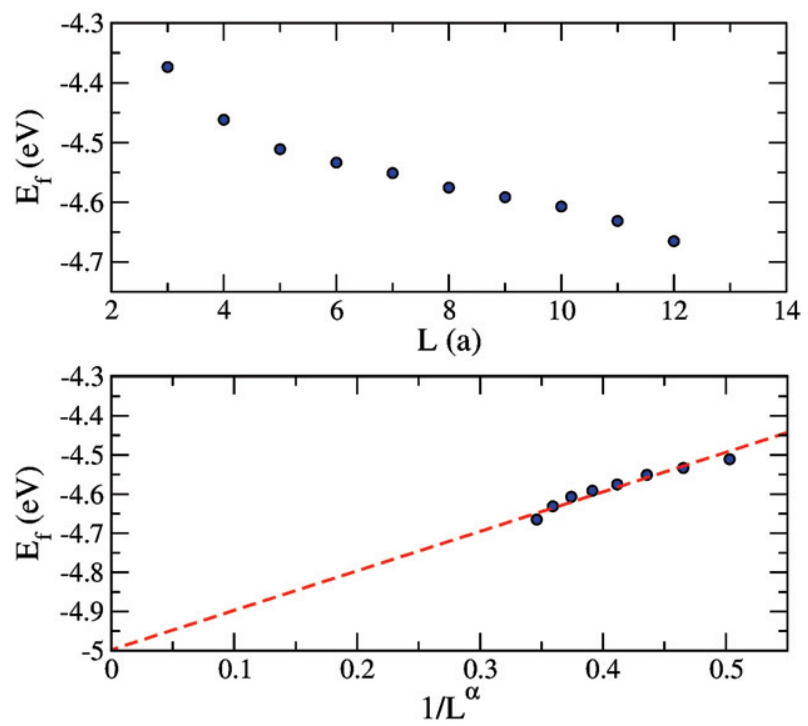

Fig. 3: (Color online) Formation energy for a carbon nanotube with boron substitutional doping as a function of (top panel) unit cell size $L$ (in units of the graphene lattice parameter, a) and (bottom panel) $1 / L^{\alpha}$, with $\alpha=0.427$. The intercept of the linear function depicted by the dashed line gives $\Delta \mathcal{E}_{1}=$ $-4.998 \mathrm{eV}$.

function of the supercell length $L$ (in units of the nanotube unit cell length, $a$ ), showing the typical behaviour of a quantity that slowly approaches convergence. Upon fitting the curve using eq. (11) we note that $\alpha<1$, which corroborates this observation. Most importantly, when plotted as a function of $1 / L^{\alpha}$ the formation energy indeed falls onto a distinctive straight line, as shown in the bottom panels of both figures. It is thus straightforward to 
obtain the formation energy through a simple extrapolation scheme, since the intercept of the line corresponds to $\Delta \mathcal{E}_{1}$.

Remarkably, the difference between the extrapolated values of $E_{\mathrm{f}}$ and those obtained from the top panels of figs. 2 and 3 can be as large as $0.2 \mathrm{eV}$. First, we note that for $L=15 a$ the total number of atoms in the system is 300. As the curve falls off very slowly, one would require a calculation with an extremely long nanotube for the changes in the formation energy to fall within $k_{\mathrm{B}} T$ at room temperature, being $k_{\mathrm{B}}$ the Boltzmann constant and $T$ the temperature.

Thus, on the one hand we have shown that significantly larger cells are required to make accurate predictions of single impurities in carbon nanotubes due to the commensurability effect. On the other hand, our deeper understanding of the effect has led to a very efficient way of obtaining the formation energy of a single dopant, a result that otherwise would be very slow to converge.

While the functional form shown in eq. (11) is confirmed by DFT results, the actual value of the exponent $\alpha$ is a lot more challenging to predict. Purely based on dimensionality arguments, we expect $\alpha$ not to be larger than a threshold. Therefore, we search for the correct exponent by fitting the formation energy results with the function shown in eq. (11) including the additional constraint that $\alpha \leq 1$. This exponent can be determined with a number of points that are associated with manageable supercell sizes.

Conclusion. - In summary, we have shown that the existing commensurability effect in the electronic structure of carbon nanostructures can be employed to derive a useful expression for the formation energy of an array of equally spaced substitutional impurities in onedimensional graphitic structures, namely a carbon nanotube. We have shown that it leads to a slow convergence of the formation energy of nitrogen and boron substitutional defects that can amount to $\sim 0.2 \mathrm{eV}$ between the typical supercell sizes used in calculations, and the result for the actual infinite sytem. We also show that the functional form of the expression allows us to manipulate the DFT calculations in order to generate accuracy levels that would otherwise require enormously large unit cells.

$$
* * *
$$

We acknowledge financial support from the Programme for Research in Third Level Institutions (PRTLI). ARR acknowledges support from FAPESP (Grant No. 2015/26862-4), ICTP-SAIRF (FAPESP project 2011/11973-4), and the ICTP-Simons Foundation Associate Scheme. MSF also acknowledges financial support from Science Foundation Ireland (Grant No. SFI 11/RFP.1/MTR/3083). Computer time was provided by IFT/Unesp, LNCC-Santos Dumont and TCHP from Trinity College Dublin.

\section{REFERENCES}

[1] Ashcroft N. W. and Mermin N. D., Solid State Physics, 1st edition (Thomson Press, India) 2003.

[2] Novoselov K. S., Geim A. K., Morozov S. V., Jiang D., Zhang Y., Dubonos S. V., Grigorieva I. V. and Firsov A. A., Science, 306 (2004) 666.

[3] Geim A. K. and Novoselov K. S., Nat. Mater., 6 (2007) 183.

[4] Iijima S., Nature, 354 (1991) 56.

[5] Lawlor J. A., Power S. R. and Ferreira M. S., Phys. Rev. B, 88 (2013) 205416.

[6] Costa A. T., Kirwan D. F. and Ferreira M. S., Phys. Rev. B, 72 (2005) 085402.

[7] Lawlor J. A. and Ferreira M. S., Beilstein J. Nanotechnol., 5 (2014) 1210.

[8] Lawlor J. A., Gorman P. D., Power S. R., Bezerra C. G. and Ferreira M. S., Carbon, 77 (2014) 645.

[9] Cheinanov V. V., Syluuasen O., Altshuler B. L. and FAL'Ko V. I., EPL, 89 (2010) 56003.

[10] Lv R., Li Q., Botello-Méndez A. R., Hayashi T., Wang B., Berkdemir A., Hao Q., Elías A. L., CruzSilva R., Gutiérrez H. R., Kim Y. A., Muramatsu H., Zhu J., Endo M., Terrones H., Charlier J.-C., PAn M. and Terrones M., Sci. Rep., 2 (2012) 1.

[11] Zabet-Khosousi A., Zhao L., Pálová L., Hybertsen M. S., Reichman D. R., Pasupathy A. N. and Flynn G. W., J. Am. Chem. Soc., 136 (2014) 1391.

[12] Lin C., Feng Y., Xiao Y., Dürr M., Huang X., Xu X., Zhao R., Wang E., Li X. Z. and Hu Z., Nano Lett., 15 (2015) 903.

[13] Wan W., Li H., Huang H., Wong S. L., Lv L., GaO Y. and WeE A. T. S., ACS Nano, 8 (2014) 970.

[14] Usachov D. Y., Fedorov A. V., Vilkov O. Y., Petukhov A. E., Rybkin A. G., Ernst A., Otrokov M. M., Chulkov E. V., Ogorodnikov I. I., Kuznetsov M. V., Yashina L. V., Kataev E. Y., Erofeevskaya A. V., Voroshnin V. Y., Adamchuk V. K., Laubschat C. and Vyalikh D. V., Nano Lett., 16 (2016) 4535.

[15] Duffy J., Lawlor J. A., Lewenkopf C. and Ferreira M. S., Phys. Rev. B, 94 (2016) 045417.

[16] Ruiz-Tijerina D. A. and DA Silva L. G. G. V. D., Phys. Rev. B, 94 (2016) 085425.

[17] Black-Schaffer A. M., Phys. Rev. B, 81 (2010) 205416.

[18] Power S. R., Guimarães F. S. M., Costa A. T., Muniz R. B. and Ferreira M. S., Phys. Rev. B, 85 (2012) 195411.

[19] Sherafati M. and Satpathy S., Phys. Rev. B, 83 (2011) 165425 .

[20] Gonzalez-Herrero H., Gomez-Rodriguez J. M., Mallet P., Moaied M., Palacios J. J., Salgado C., Ugeda M. M., Veuillen J.-Y., Yndurain F. and Brihuega I., Science, 352 (2016) 437.

[21] Venezuela P., Muniz R. B., Costa A. T., Edwards D. M., Power S. R. and Ferreira M. S., Phys. Rev. $B, 80$ (2009) 241413.

[22] Zanolli Z. and Charlier J.-C., Phys. Rev. B, 81 (2010) 165406.

[23] Hohenberg P. and Kohn W., Phys. Rev., 136 (1964) B864. 
[24] Kohn W. and Sham L., Phys. Rev., 144 (1965) A1133.

[25] Lawlor J. A. and Ferreira M. S., Phys. Rev. B, 92 (2015) 115405.

[26] Kresse G. and Furthmüller J., Phys. Rev. B, 54 (1996) 11169.

[27] Giannozzi P., Baroni S., Bonini N., Calandra M., Car R., Cavazzoni C., Ceresoli D., Chiarotti G. L., Cococcioni M., Dabo I., Dal Corso A., de Gironcoli S., Fabris S., Fratesi G., Gebauer R., Gerstmann U., Gougoussis C., Kokalu A., Lazzeri M., Martin-Samos L., Marzari N., Mauri F., Mazzarello R., Paolini S., Pasquarello A., Paulatto L., Sbraccia C., Scandolo S., Sclauzero G., Seitsonen A. P., Smogunov A., Umari P. and Wentzcovitch R. M., J. Phys.: Condens. Matter, 21 (2009) 395502.
[28] Soler J. M., Artacho E., Gale J. D., García A., Junquera J., Ordejón P. and SÁnchez-Portal D., J. Phys.: Condens. Matter, 14 (2002) 2745.

[29] Padilha J. E., Amorim R. G., Rocha A. R., Da Silva A. J. R. and FAzzio A., Solid State Commun., 151 (2011) 482.

[30] Rocha A. R., Padilha J., Fazzio A. and Da Silva A. J., Phys. Rev. B, $7 \mathbf{7}$ (2008) 153406.

[31] Lambin P., Amara H., Ducastelle F. and Henrard L., Phys. Rev. B, 86 (2012) 045448.

[32] Khalfoun H., Lherbier A., Lambin P., Henrard L. and Charlier J.-C., Phys. Rev. B, 91 (2015) 035428.

[33] Perdew J. P., Burke K. and Ernzerhof M., Phys. Rev. Lett., 77 (1996) 3865.

[34] Troullier N. and Martins J. L., Phys. Rev. B, 43 (1991) 1993. 